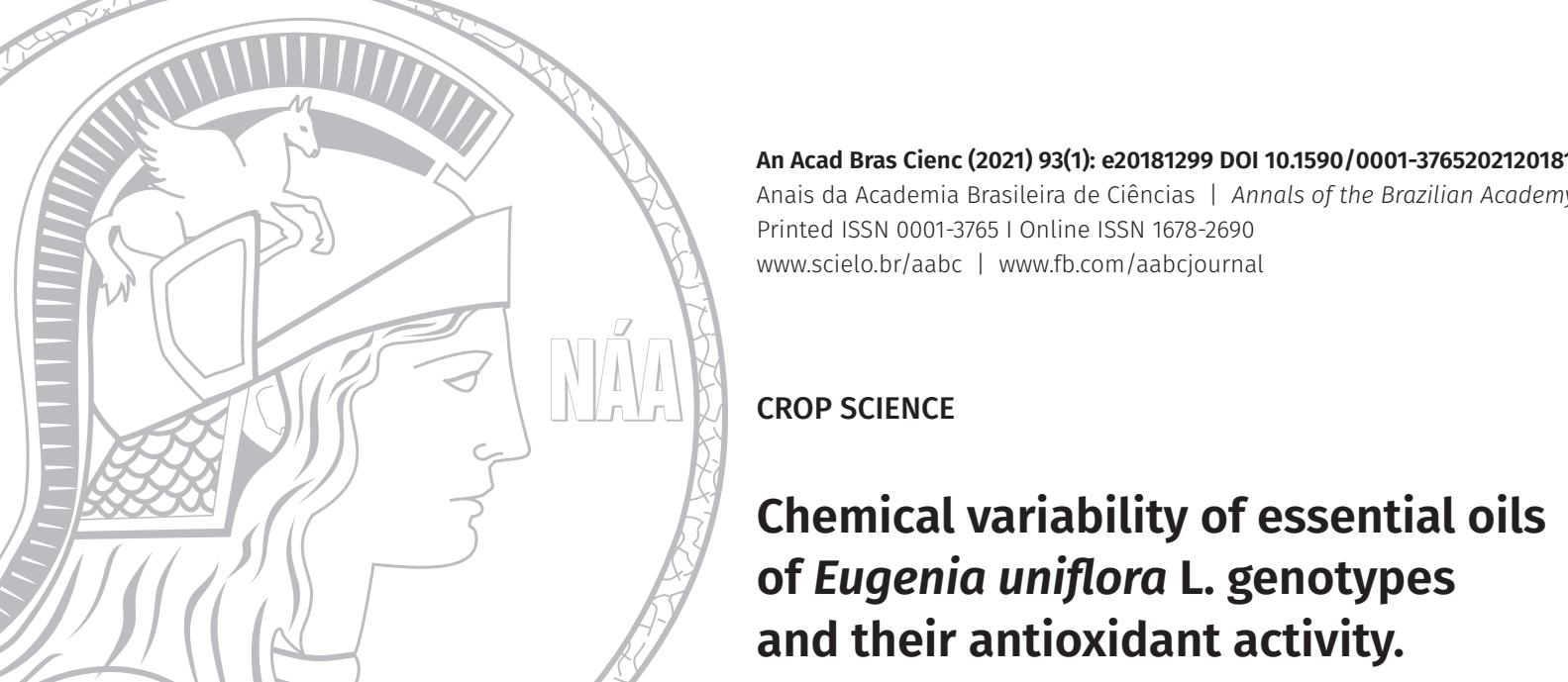

\title{
Chemical variability of essential oils of Eugenia uniflora L. genotypes and their antioxidant activity.
}

\author{
ROGER R. CIPRIANO, BEATRIZ H.L.N.S. MAIA \& CÍCERO DESCHAMPS
}

\begin{abstract}
Eugenia uniflora, known as the "Brazilian cherry", is an economically important neotropical Myrtaceae in the cosmetics and pharmaceutical industries due the production of essential oils with antioxidant activity. On account of its significant genetic variability, genotype evaluations are needed in order to identify genetic features related to the essential oil production that meet the industry requirements. The main objective of the present study was to evaluate the yield, composition, and antioxidant activity of essential oils isolated from the leaves of 36 genotypes of E. uniflora. Essential oil samples were obtained by hydrodistillation, and their composition was determined by gas chromatography coupled with mass spectrometry. A variation of $0.22 \%$ to $1.68 \%$ in the essential oil yield was observed, in which 78 compounds, namely oxygenated sesquiterpenes, were identified. According to the cluster analysis of the major compounds, six groups were revealed. The observed diversity demonstrates the genetic variability of the species. Also, the antioxidant activity was affected by the composition of the essential oils, ranging from 176.66 to $867.57 \mu \mathrm{M}$ TEAC.
\end{abstract}

Key words: DPPH, genetic diversity, Myrtaceae, sesquiterpenes.

\section{INTRODUCTION}

Eugenia uniflora L., popularly known as the "Brazilian cherry" or "Pitangueira", is one of the neotropical species of the Myrtaceae family employed in the food and cosmetics industries.

Until recently, the species had been utilized in fruit production on account of its exotic flavors and aromas, as well as its high pulp yield and nutritional value. The fruit's pulp is used in the production of juice, ice cream, jellies, and liqueurs, or commercialized in natura or frozen. According to Oliveira et al. (2006), its juice is rich in vitamins and antioxidant compounds.

E. uniflora presents ducts and secretory cavities in its mesophyll (Amstrong et al. 2012, Retamales \& Scharaschkin 2015, Pacheco-Silva \& Donato 2016), where oil resins and terpene compounds are synthesized (Thadeo et al. 2009, Stesevic et al. 2016, Bomboa et al. 2017). The plant's essential oil, composed primarily of terpenes, is utilized by pharmaceutical and cosmetics industries because of its antioxidant activity (Victoria et al. 2012), which can be used against human tumor cells, as demonstrated by Ogunwande et al. (2005), while working with Leishmania amazonensis (Rodrigues et al. 2013, Silva et al. 2018).

The chemical constitution of the aromatic species' essential oils exhibits considerable variability due to environmental factors. E. uniflora leaves produce essential oils with distinct chemical compositions according to the different seasons of the year, altering the percentage of sesquiterpenes between the humid and dry periods (Costa et al. 2009). Such 
composition variability in the species was also verified in genotypes collected in different regions of Brazil. The major compounds present in the plants collected in the Brazilian cerrado (Silva et al. 2018) were germacrone (8.52\%) and $\alpha$-selinene (7.50\%). Meanwhile, the oil from plants collected in the state of Ceará was composed primarily of Selina-1,3,7(11)-trien-8one (36.37\%) and Selina-1,3,7(11)-trien-8-one epoxide (27.32\%) (Santos et al. 2018), whereas in Maranhão, the predominant constituent was curzerene (47.30\%) (Rodrigues et al. 2013).

Another reason for the variation in the chemical composition of the species' essential oils is genetic. According to Santos et al. (2015), essential oil from young leaves presented higher percentages of curzerene (22.37\%) than mature leaves (16.60\%), and furanodiene was not found in mature leaves, nor was germacrone in young leaves. When evaluating the correlation between the chemical composition of essential oils from different colored fruits, Costa et al. (2010) identified three different chemotypes. The compound curzerene was absent in plants whose fruits were orange, although present in $20.50 \%$ in plants with yellow fruits and $42.60 \%$ in plants with red fruits.

Thus, the present study aimed at evaluating the yield and chemical composition of essential oil from leaves of E. uniflora L. genotypes and their antioxidant activity.

\section{MATERIALS AND METHODS}

\section{Plant material}

The plant material was collected from 36 genotypes, aged 11 years old, obtained from seedlings of four plants cultivated in the Experimental Farm of the Federal University of Paraná, Pinhais - PR, Brazil, located at approximately $25^{\circ} 23^{\prime} 30^{\prime \prime} \mathrm{S}, 4^{\circ} 07^{\prime} 30^{\prime \prime} \mathrm{W}$, in March 2017.

\section{Essential oil extractions and analysis}

A total of $400 \mathrm{~g}$ of leaves were dried at room temperature during 48 hours for essential oil isolation (Assis et al. 2020). The oil samples were obtained by hydrodistillation in a Clevenger apparatus for 4 hours in three repetitions, each one containing $100 \mathrm{~g}$ of leaves with $1000 \mathrm{~mL}$ of distilled water. The essential oil yield was determined based on dry mass and expressed as a percentage $\left(\% \mathrm{~m} \cdot \mathrm{m}^{-1}\right)$.

The extracted oil was stored in a freezer at $-20^{\circ} \mathrm{C}$ until analysis. In order for the identification and quantification of the essential oil components, the samples were diluted in hexane until an oil concentration of $1 \%$. A $1.0 \mu \mathrm{L}$ aliquot of such solution was injected into a gas chromatograph coupled with a mass spectrometer (GC/MS) (Shimadzu 2010 Plus) - UFPR Chemistry Department, using an injector maintained at $250^{\circ} \mathrm{C}$. Separation of the constituents was performed using an HP5MS capillary column $(30 \mathrm{~m} \times 0.25 \mathrm{~mm} \times 0.25$ $\mu \mathrm{m})$ with helium gas as a carrier $\left(1.0 \mathrm{~mL} \cdot \mathrm{min}^{-1}\right)$. The oven temperature was programmed to gradually increase from $60^{\circ} \mathrm{C}$ to $240^{\circ} \mathrm{C}$ at a rate of $3^{\circ} \mathrm{C} \cdot \mathrm{min}^{-1}$. Chemical constituent identification was conducted by comparing their mass spectra with a database, and also their linear retention indices, calculated by the injection of a homologous series of $n$-alkanes (Van Den Dool \& Kratz 1963), which were compared with data available in the literature (Adams 2007). Meanwhile, for compound quantification, a GC coupled with a flame ionization detector (FID) was used, in the same conditions described above, with the exception of the carrier gas (hydrogen, at $1.5 \mathrm{~mL} \cdot \mathrm{min}^{-1}$ ). The percentage composition, in turn, was obtained by the electronic integration of the FID signal, by dividing the area of each component by the total area (\%). 


\section{Antioxidant activity (AA)}

The antioxidant activity of the essential oils was determined by measuring the amount of the free radical DPPH (2,2-diphenyl-1-picrylhydrazyl), according to the procedure described by BrandWilliams et al. (1995). The oils were diluted in methanol 1:5(20\%) to a final volume of $100 \mu \mathrm{L}$ and added to $3.9 \mathrm{~mL}$ of DPPH solution in methanol $\left(23.66 \mu \mathrm{g} \cdot \mathrm{mL}^{-1}\right)$. The reaction was performed in the dark and at room temperature for 30 minutes, and the absorbances were read using a UV/VIS Shimadzu ${ }^{\oplus}-1800$ spectrophotometer, at $515 \mathrm{~nm}$. Trolox (6-hydroxy-2,5,7-tetramethylchroman2-carboxylic acid) was used as a synthetic reference, and the analyses were conducted in triplicate. The results were expressed as Trolox Equivalent Antioxidant Capacity (TEAC), in $\mu \mathrm{M}$.

\section{Statistical analysis}

In order to measure the essential oil yield and antioxidant activity, the variances were tested for homogeneity by the Bartlett test, and the means were compared using the Scott-Knott test $(p<0.05)$. The statistical analysis was carried out with the ASSISTAT program version 7.7 (Silva \& Azevedo 2016).

The hierarchical cluster analysis method was performed using the R software, version 3.4.1 (R Core Team 2014), to determine the chemical similarity of the genotypes. In this analysis, 15 major compounds were included, with a percentage greater than $5 \%$ in at least one of the genotypes (Table II).

\section{RESULTS AND DISCUSSION}

\section{Essential oil yield and composition}

All of the analyzed E. uniflora genotypes produced essential oil, with yields ranging from 0.22 to $1.68 \%$ (average of $0.62 \%$ ) (Figure 1 ; Table I). The genotypes A05, A06, A11, A15, A18, A31, and A36 presented essential oil yields above $1 \%$ on average.

The average essential oil yield was higher than those reported in other studies performed with the species. Galhiane et al. (2006) obtained a yield equivalent to $0.55 \%$ from dried leaves. Rodrigues et al. (2013), in turn, reported a 0.30\%

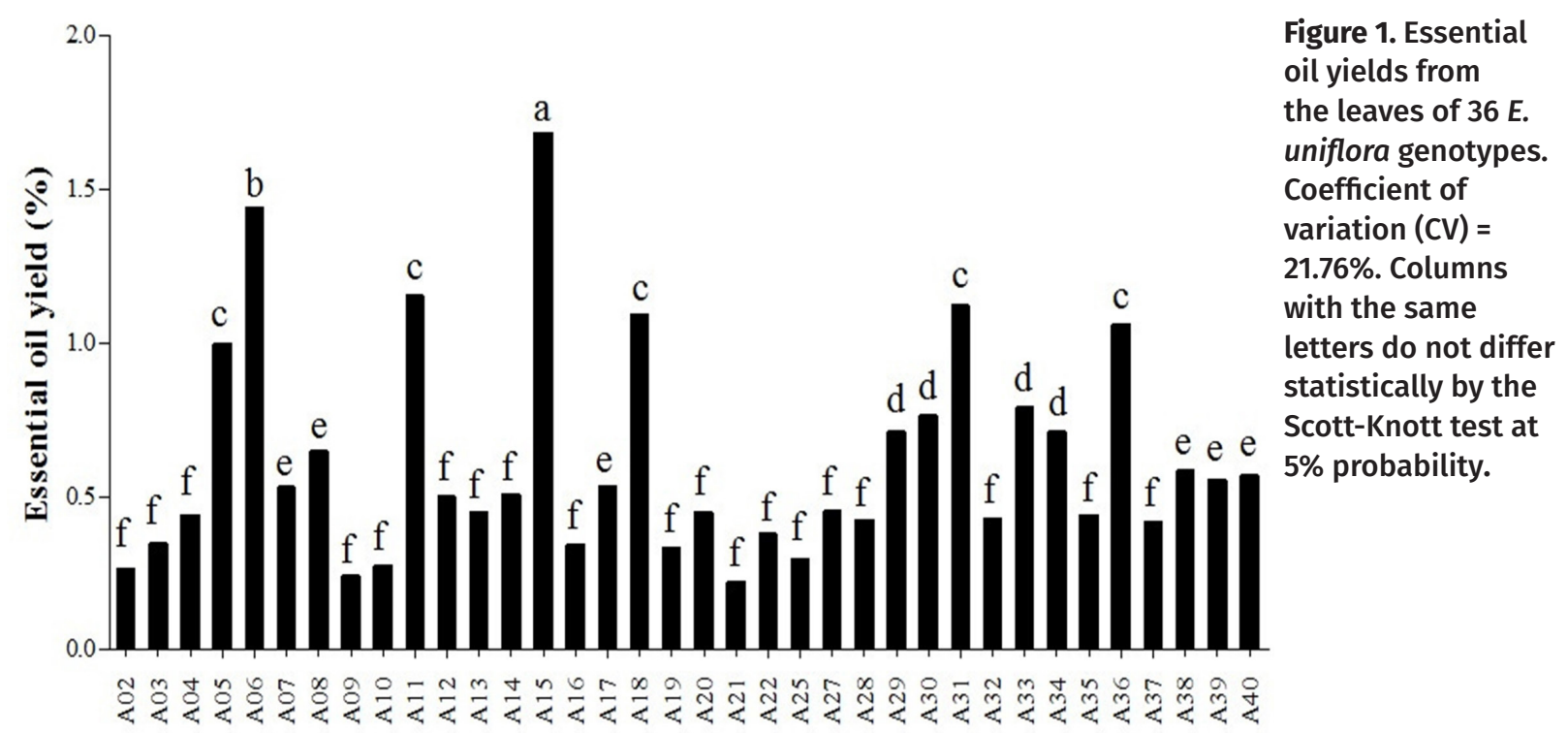

\section{Genotypes}


Table I. Essential oil yield, identified compounds, hydrocarbon and oxygenated monoterpenes, and hydrocarbon and oxygenated sesquiterpenes, observed in the analyzed E. uniflora genotypes.

\begin{tabular}{|c|c|}
\hline & Means ( \pm SD) \\
\hline Essential Oil yield (\%) & $0.62( \pm 0.34)$ \\
\hline Identified compounds (\%) & $67.26( \pm 15.67)$ \\
\hline Monoterpene hydrocarbons (\%) & $0.31( \pm 0.76)$ \\
\hline Oxygenated monoterpenes (\%) & $0.00( \pm 0.00)$ \\
\hline Sesquiterpene hydrocarbons (\%) & $26.05( \pm 9.17)$ \\
\hline Oxygenated sesquiterpenes (\%) & $40.90( \pm 20.17)$ \\
\hline
\end{tabular}

SD = Standard deviation.

yield, whereas Silva et al. (2018) found an average essential oil yield of $0.51 \%$ in E. uniflora.

The activation of biosynthetic routes of terpene compounds is influenced by changes in environmental conditions (Verna \& Shukla 2015). Plants subjected to the same conditions, as in the present study, vary due to genetic characteristics.

A total of 78 compounds were identified in the essential oil samples (Table SIV Supplementary Material), with an average of

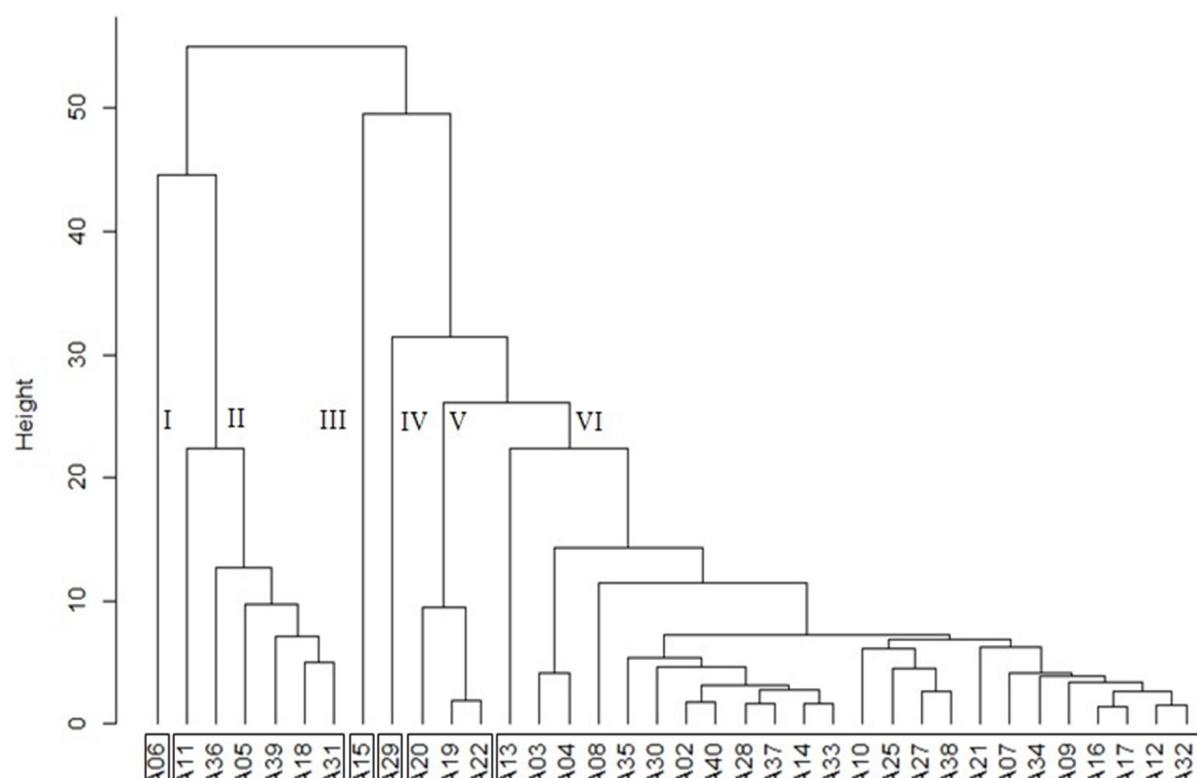

$67.26 \%$ of the identified compounds present in the genotypes. Most of the constituents were oxygenated sesquiterpenes (Table I), corroborating with findings described by other authors (Costa et al. 2009, Gallucci et al. 2010, Chang et al. 2011, Rodrigues et al. 2013).

According to Stefanello et al. (2011), sesquiterpene compounds, predominant in most species of the Myrtaceae family, are responsible for their biological properties. Essential oils from these species containing such compounds have demonstrated potent antioxidant activity (Theanphong et al. 2015), antimicrobial and antiparasitic properties (Lago et al. 2011, Sousa et al. 2015, Araújo et al. 2017, Ghazouani et al. 2017), inhibitory action on tumor cell growth (Liu et al. 2013, Zhong et al. 2016, Pereira et al. 2017), and antihypertensive effects (Kumar et al. 2017).

The essential oil analysis of the 36 genotypes using the cluster analysis method enabled the identification of six groups associated with different chemical compositions (Figure 2; Figure 3).

Groups I, III, and IV were composed of only one access each, the first of which presented germacrene B (9.27\%), Selina-1,3,7(11)-trien-8-one

Figure 2. Cluster analysis based on the essential oil main constituents of 36 genotypes of $E$. uniflora, obtained by the UPGMA method. Cophenetic correlation $=95.88 \%$. 
(29.41\%), germacrone (18.58\%), and Selina1,3,7(11)-trien-8-one epoxide (9.07\%) as primary compounds. The essential oil of group III was composed mainly of curzerene (9.18\%), 7-epi$\alpha$-selinene $(28.00 \%), \delta$-cadinene $(9.90 \%)$, epi$\alpha$-muurolol (10.25\%), and $\alpha$-cadinol (16.98\%). The major compounds found in group IV, in turn, were curzerene (18.45\%), germacrene B (9.40\%), $\mathrm{C}_{15} \mathrm{H}_{24} \mathrm{O}$ (12.22\%), germacrone (9.15\%), and furanodiene (8.36\%).

Group II, on the other hand, consisted of six genotypes, composed mainly of curzerene (7.95 $13.01 \%)$, germacrene $\mathrm{B}(2.18-11.36 \%), \mathrm{C}_{15} \mathrm{H}_{24} \mathrm{O}(1.46$ - 11.39), and germacrone (35.26 - 65.03\%). Group $\checkmark$ was represented by three major genotypes: germacrene B $(4.77-6.36 \%), \mathrm{C}_{15} \mathrm{H}_{24} \mathrm{O}(12.78$ 17.57\%), and 7,14-anhydro-amorpha-4,9-diene (13.65 - 22.16\%). A total of 24 genotypes were included in group $\mathrm{VI}$, whose primary compounds comprised bicyclogermacrene (1.39 - 8.39\%), germacrene $\mathrm{B}(8.43-19.75 \%)$, and $\mathrm{C}_{15} \mathrm{H}_{24} \mathrm{O}(13.60$ $-46.13 \%)$.

The chemical diversity of an essential oil can be due to environmental conditions, such as the collection site (Zoghbia et al. 2011, Victoria et al. 2012, Rezende et al. 2013), season of the year (Costa et al. 2009, Ferreira et al. 2017), and age of the leaves and plants (Santos et al. 2015).
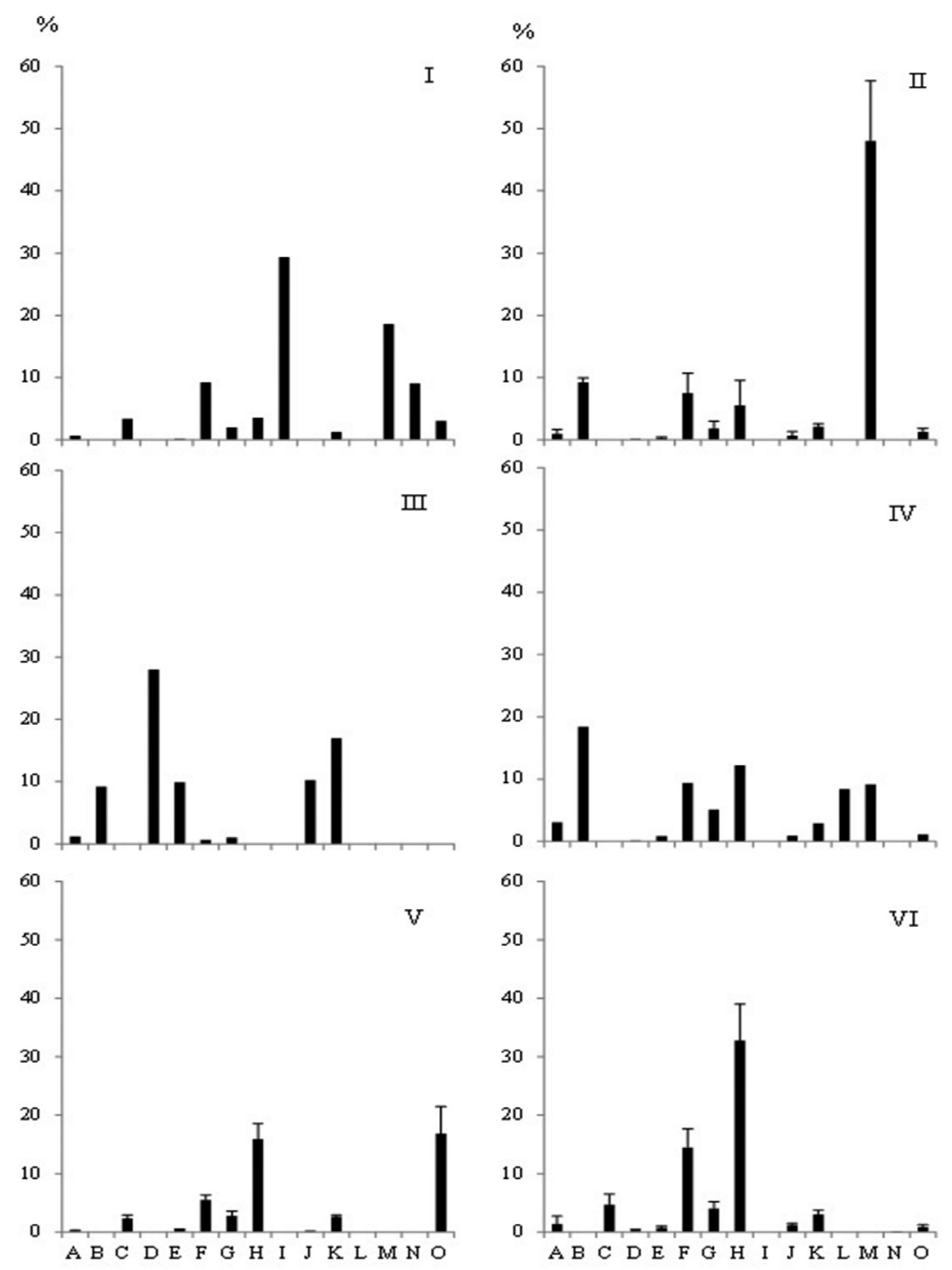

Figure 3. Primary essential oil constituents of the six groups of $E$. uniflora genotypes. Values are expressed as mean \pm SD (standard deviation). A germacrene D; B curzerene; C bicyclogermacrene; D 7-epi- $\alpha$-selinene; $\mathrm{E}$ $\delta$-cadinene; F germacrene B; G thusopsan-2 $\alpha$-ol; $\mathrm{H}$ $\mathrm{C}_{15} \mathrm{H}_{24} \mathrm{O}$; I selina-1,3,7(11)trien-8-one; J epi- $\alpha$ muurolol; K $\alpha$-cadinol; L furanodiene; M germacrone; N selina-1,3,7(11)trien-8-one epoxide; 0 7,14-anhydro-amorpha-4,9diene. 


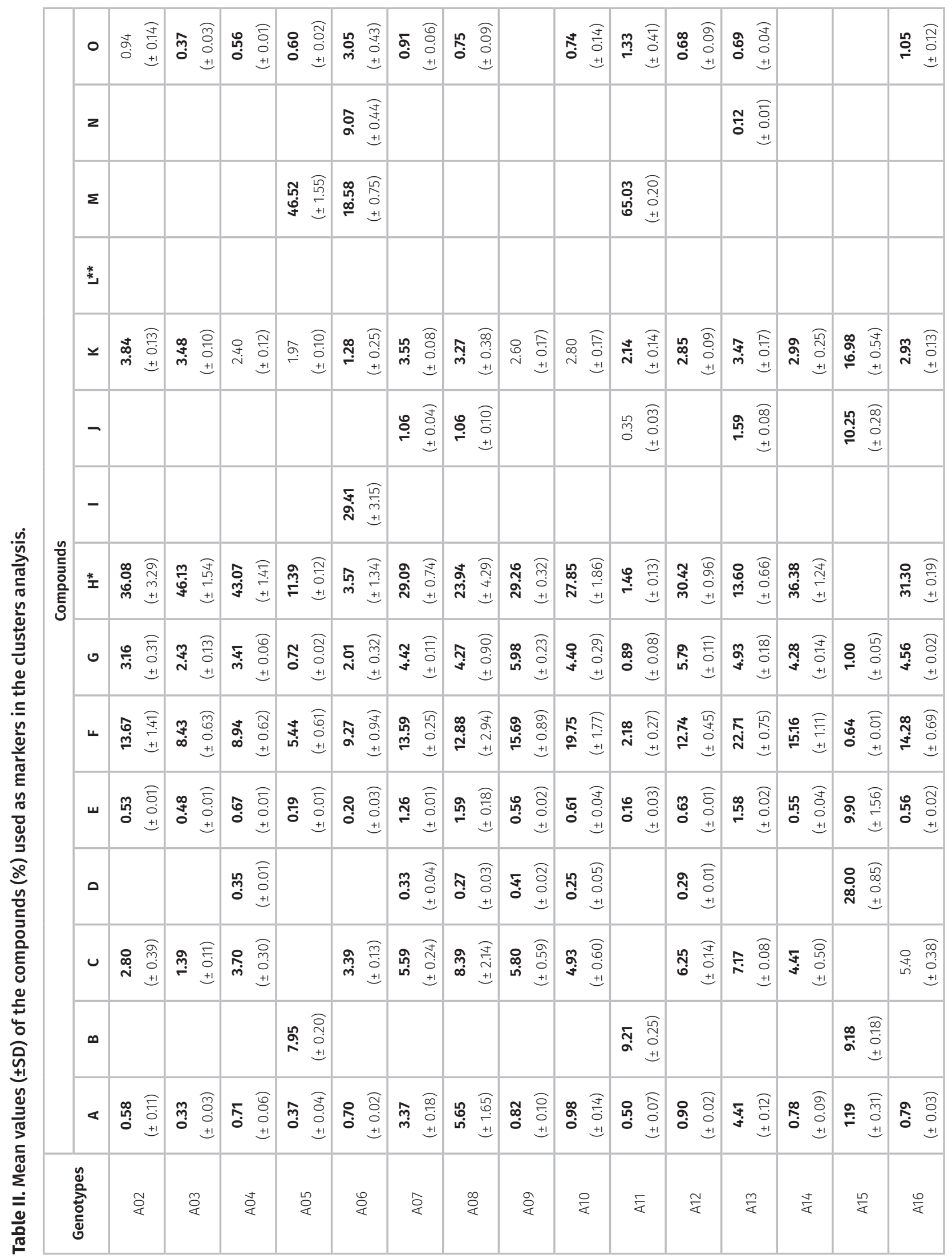




\begin{tabular}{|c|c|c|c|c|c|c|c|c|c|c|c|c|}
\hline $\mid \begin{array}{cc}\widehat{\pi} \\
\mathfrak{a} & 0 \\
& +1\end{array}$ & 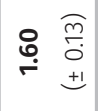 & 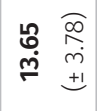 & 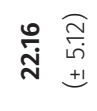 & 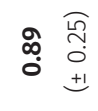 & 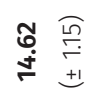 & 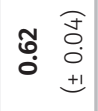 & 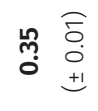 & $\begin{array}{cc} & \widehat{\sigma} \\
\infty & 0 \\
0 & 0 \\
0 & \pm 1\end{array}$ & 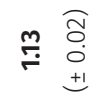 & 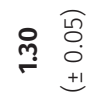 & 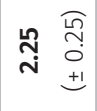 & $\begin{array}{cc}0 & \widehat{0} \\
0 & 0 \\
0 & 0 \\
0 & +1\end{array}$ \\
\hline & 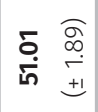 & & & & & & & & 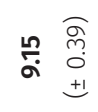 & & 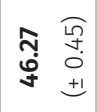 & \\
\hline & & & & & & & & & 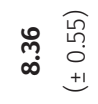 & & & \\
\hline 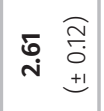 & 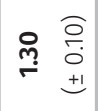 & 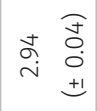 & 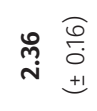 & 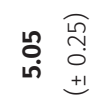 & 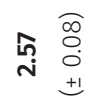 & 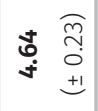 & 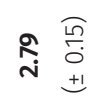 & 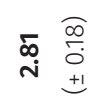 & 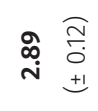 & 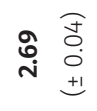 & & 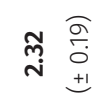 \\
\hline & $\begin{array}{cc}\widetilde{0} & \widehat{\delta} \\
\stackrel{0}{0} & 0 \\
0 & +1\end{array}$ & & $\begin{array}{cc}\infty \\
\stackrel{\infty}{0} \\
0 \\
0 \\
0 \\
+1\end{array}$ & 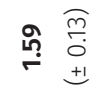 & & 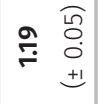 & & & $\begin{array}{ll}\widehat{O} \\
0 \\
0 \\
0 \\
0 \\
\\
\\
\pm 1\end{array}$ & & $\begin{array}{ll}\bar{m} & \widehat{o} \\
0 \\
0 \\
0 \\
\pm 1\end{array}$ & \\
\hline 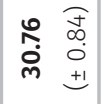 & 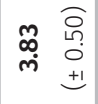 & $\begin{array}{ll}\sqrt[n]{n} & \widehat{6} \\
\stackrel{0}{\circ} & +1\end{array}$ & 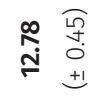 & 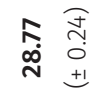 & 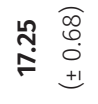 & 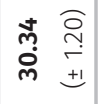 & 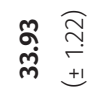 & 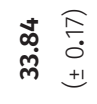 & 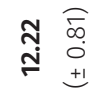 & 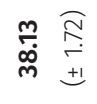 & 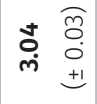 & 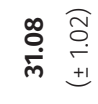 \\
\hline 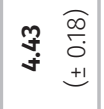 & 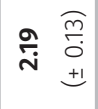 & $\begin{array}{ll}\stackrel{O}{0} \\
\stackrel{0}{\circ} \\
\stackrel{+}{ \pm}\end{array}$ & 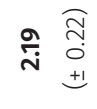 & 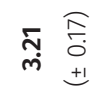 & 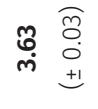 & $\begin{array}{c}\widehat{a} \\
\stackrel{0}{0} \\
\stackrel{0}{\circ} \\
+1\end{array}$ & 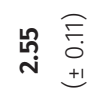 & 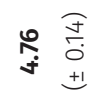 & 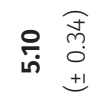 & 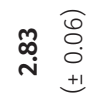 & 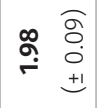 & 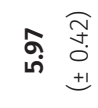 \\
\hline 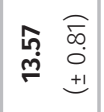 & $\bar{c} \begin{array}{c}\widehat{\sigma} \\
0 \\
0 \\
0 \\
+1\end{array}$ & 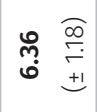 & 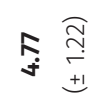 & 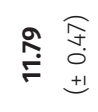 & 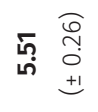 & 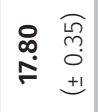 & 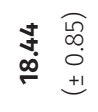 & 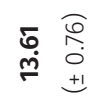 & 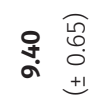 & 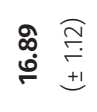 & 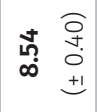 & 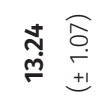 \\
\hline $\begin{array}{cc}\tilde{5} & \widehat{0} \\
0 & 0 \\
0 & \pm \\
& \pm 1\end{array}$ & 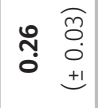 & 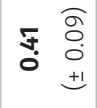 & 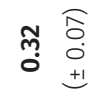 & $\bar{m} \begin{array}{c}\widehat{O} \\
0 \\
0 \\
+1\end{array}$ & 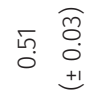 & 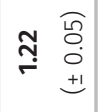 & $\overline{\sigma o} \begin{array}{c}\widehat{o} \\
0 \\
0 \\
0 \\
+1\end{array}$ & 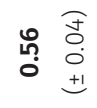 & 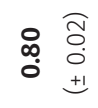 & \begin{tabular}{ll}
$\infty$ & $\bar{\sigma}$ \\
\multirow{0}{*}{} & 0 \\
0 & 0 \\
& +1
\end{tabular} & 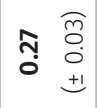 & $\begin{array}{ll}\Re & \widehat{o} \\
0 & 0 \\
0 & 0 \\
+1\end{array}$ \\
\hline $\begin{array}{cc}\overline{\tilde{n}} & \overline{0} \\
0 & 0 \\
& \pm 1\end{array}$ & & & & & & 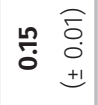 & $\begin{array}{ll}\bar{f} & \widehat{\sigma} \\
0 \\
0 \\
0 \\
\pm 1\end{array}$ & 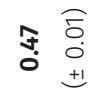 & $\begin{array}{cc}\bar{\sigma} \\
\dot{0} \\
0 \\
+1\end{array}$ & 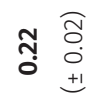 & & $\begin{array}{cc}\stackrel{0}{0} \\
0 \\
0 \\
0 \\
0 \\
+1\end{array}$ \\
\hline 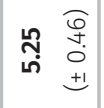 & & 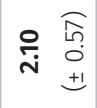 & 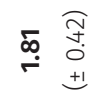 & 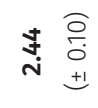 & 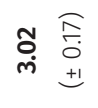 & 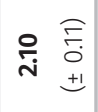 & 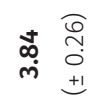 & $\begin{array}{cc}\infty & \widehat{o} \\
\infty & 0 \\
\dot{0} & 0 \\
+ & +1\end{array}$ & & 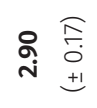 & & 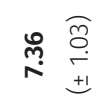 \\
\hline & 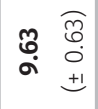 & & & & & & & & 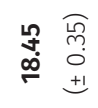 & & 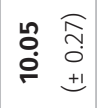 & \\
\hline $\begin{array}{cc}n & \widehat{\sigma} \\
& 0 \\
0 & 0 \\
& \pm\end{array}$ & 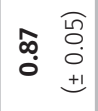 & 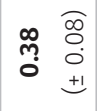 & 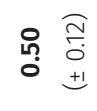 & 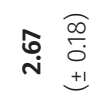 & 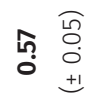 & 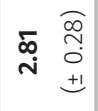 & 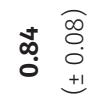 & 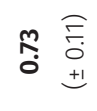 & 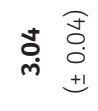 & 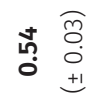 & 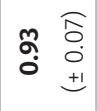 & 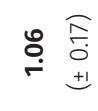 \\
\hline$\hat{\varepsilon}$ & $\underset{\leftarrow}{\infty}$ & $\stackrel{g}{\dot{z}}$ & ষ্ণ & $\bar{q}$ & $\tilde{\gtrless}$ & $\stackrel{\vec{\chi}}{\mathbb{\alpha}}$ & $\dot{\mathcal{Z}}$ & $\stackrel{\infty}{\underset{\leftarrow}{\Psi}}$ & 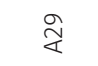 & ఖ్ & 安 & $\ddot{\dot{z}}$ \\
\hline
\end{tabular}




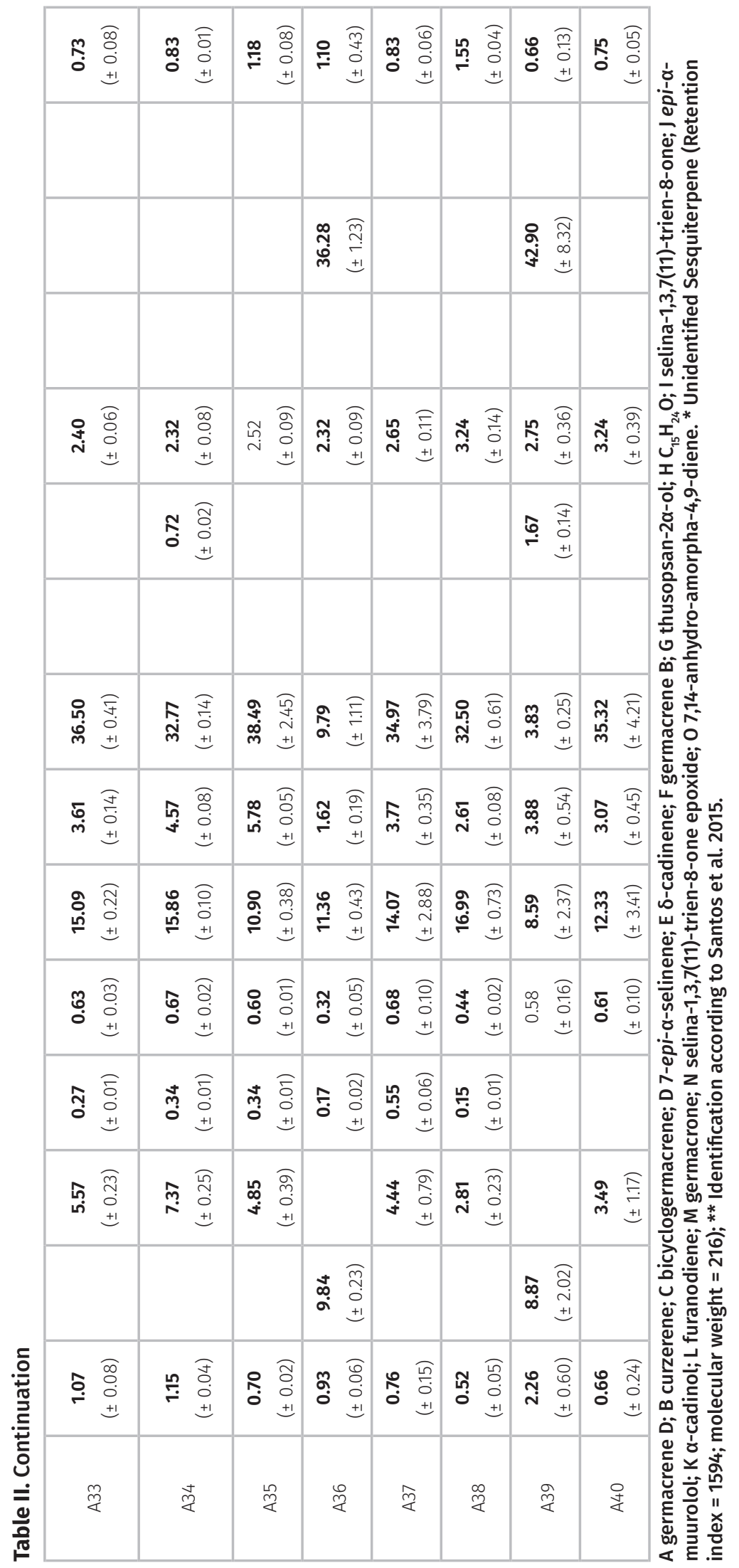


Table III. Antioxidant activity of essential oils extracted from leaves of different genotypes of $E$. uniflora by the DPPH method.

\begin{tabular}{|c|c|c|c|}
\hline Genotypes & TEAC $\pm S D(\mu M)$ & Genotypes & $\mathrm{TEAC} \pm \mathrm{SD}(\mu \mathrm{M})$ \\
\hline $\mathrm{A} 02$ & $679.69 \pm 54.55^{d}$ & $\mathrm{~A} 20$ & $464.54 \pm 60.53^{f}$ \\
\hline $\mathrm{A} 03$ & $516.05 \pm 72.73^{f}$ & $\mathrm{~A} 21$ & $655.45 \pm 41.99^{d}$ \\
\hline $\mathrm{A} 04$ & $461.51 \pm 54.55^{f}$ & A22 & $552.42 \pm 48.10^{e}$ \\
\hline A05 & $867.57 \pm 20.99^{a}$ & A25 & $509.99 \pm 20.99^{f}$ \\
\hline A06 & $231.21 \pm 27.77^{9}$ & A27 & $491.81 \pm 20.99^{f}$ \\
\hline $\mathrm{A} 07$ & $582.72 \pm 20.99^{e}$ & A28 & $643.33 \pm 72,73^{d}$ \\
\hline $\mathrm{A} 08$ & $576.66 \pm 45.76^{\mathrm{e}}$ & A29 & $843.33 \pm 26,76^{a}$ \\
\hline A09 & $606.96 \pm 65.66^{e}$ & $\mathrm{~A} 30$ & $503.93 \pm 63.85^{f}$ \\
\hline A10 & $576.66 \pm 41.99^{e}$ & A31 & $546.36 \pm 75.70^{\mathrm{e}}$ \\
\hline $\mathrm{A} 11$ & $861.51 \pm 26.96^{a}$ & A32 & $716.05 \pm 62.98^{c}$ \\
\hline $\mathrm{A} 12$ & $764.54 \pm 27.77^{b}$ & A33 & $697.87 \pm 18.18^{c}$ \\
\hline A13 & $643.33 \pm 65.56^{d}$ & A34 & $570.60 \pm 36.36^{e}$ \\
\hline A14 & $497.87 \pm 5.33^{f}$ & A35 & $655.45 \pm 63.85^{d}$ \\
\hline A15 & $176.66 \pm 41.99^{9}$ & A36 & $588.78 \pm 72.73^{\mathrm{e}}$ \\
\hline A16 & $643.33 \pm 48.10^{d}$ & A37 & $740.30 \pm 55.55^{c}$ \\
\hline A17 & $734.24 \pm 18.18^{c}$ & A38 & $516.05 \pm 48.10^{f}$ \\
\hline A18 & $522.12 \pm 10.50^{f}$ & A39 & $782.72 \pm 27.77^{b}$ \\
\hline A19 & $709.99 \pm 45.76^{c}$ & $\mathrm{~A} 40$ & $464.54 \pm 60.53^{f}$ \\
\hline
\end{tabular}

Values are expressed as mean TEAC (Trolox equivalent antioxidant capacity) \pm SD (standard deviation) in $\mu$ M. Same letters do not differ statistically by the Scott-Knott test at $5 \%$ probability. Coefficient of variation (CV) $=8.27 \%$.

Costa et al. (2010) verified variability in the essential oil composition of "Brazilian cherry" leaves regarding the fruit color of the genotype. According to Griffis \& Manners (2005), the inheritance concerning the fruit color of E. uniflora is determined by a single gene. Therefore, the correlation observed by Costa et al. (2010) may indicate that, in addition to environmental conditions, the essential oil composition can be determined genetically.

The genetic variability of the yield and composition of the essential oils obtained from the analyzed genotypes may be related to the preferred form of reproduction of the species. According to Silva \& Pinheiro (2007), E. uniflora presents intermediate sexual reproduction; that is, both self-fertilization and cross-fertilization may occur. Nonetheless, allogamy is its preferred mode of reproduction, which is facilitated by its main pollinating agent, Apis mellifera (Diniz \& Buschini 2016), thus promoting the genetic variability of the species.

\section{Antioxidant activity}

The essential oils isolated from all genotypes of E. uniflora were positive for antioxidant activity by the DPPH inhibition method (Table III), which ranged from 176.66 to $867.57 \mu \mathrm{M}$ TEAC. Genotypes A05, A11, and A29 presented more than $800 \mu \mathrm{M}$ TEAC, statistically higher than the other genotypes, and were composed primarily of curzerene, furanodiene, and germacrone. 
Studies with species of the genus Curcuma have demonstrated that such compounds isolated from their essential oils exhibited strong antioxidant activity (Zhao et al. 2010, Hamdi et al. 2015) and, therefore, may be responsible for the high sequestration rate of DPPH.

The genotypes whose main essential oil compounds were germacrone and curzerene (A18, A31, and A39) did not present the same DPPH free radical inhibition performance. Those composed only of curzerene (A15) or germacrone (A06) also showed little effect on the free radical. Other compounds, which were not observed and/or were in lower percentages in the essential oils that showed higher antioxidant activity, were also found in the three genotypes, including Selina-1,3,7(11)trien-8-one (A06) and 7-epi- $\alpha$-selinene (A15). According to Wang et al. (2008), it is difficult to attribute an antioxidant effect to one or a few compounds present in essential oils since they are considered a complex mixture of chemical components and, therefore, the interaction between them, including minority compounds, can influence such effects (Botelho et al. 2007). Thus, the association between all of the compounds present in the oil are fundamental for the sequestration of this free radical.

\section{CONCLUSIONS}

The genotypes of the "Brazilian cherry" showed significant genetic variability regarding essential oil yield and composition. Six groups were identified according to chemical constitution, in which Selina-1,3,7(11)-trien-8-one, Selina1,3,7(11)-trien-8-one epoxide, curzerene, germacrone, 7-epi-a-selinene, furanodiene, 7,14-anhydro-amorpha-4,9-diene, and $\mathrm{C}_{15} \mathrm{H}_{24} \mathrm{O}$ were the main compounds that differentiated the groups. Most of the E. uniflora essential oil constituents found in the present study were sesquiterpenes (94.45\%), a fact that may have influenced in the antioxidant activity of the analyzed oils. The variability of the essential oil composition and the amplitude in the antioxidant effect reinforce the need for quality control of the oils used in the pharmaceutical and cosmetics industries.

\section{Acknowledgments}

The authors acknowledge the Coordenação de Aperfeiçoamento de Pessoal de Nivel Superior (CAPES) for financial support.

\section{SUPPLEMENTARY MATERIAL}

\section{Table SIV.}

\section{REFERENCES}

ADAMS RP. 2007. Identification of essential oil components by gas chromatography/ mass spectrometry. 4(1) Ed., Carol Stream, Allured Publishing Corporation, Illinois, 804 p.

AMSTRONG L, DUARTE MR \& MIGUEL OG. 2012. Morphoanatomy of the leaf and stem of Eugenia pyriformis. Rev Bras Farmacogn 22(3): 475-481.

ARAÚJO FM, DANTASA MCSM, SILVA LS, AONA LYS, TAVARES IF \& SOUZA-NETAC LC. 2017. Antibacterial activity and chemical composition of the essential oil of Croton heliotropiifolius Kunth from Amargosa, Bahia. Brazil. Ind Crop Prod 105: 203-206.

ASSIS ALA, CIPRIANO RR, CUQUEL FL \& DESCHAMPS C. 2020. Effect of drying method and storage conditions on the essential oil yield and composition of Eugenia uniflora L. leaves. Rev Colomb Cienc Hort 14(2): 275-282.

BOMBOA AB, FILARTIGA AL, GARCIA VL \& APPEZZATO-DAGLÓRIA B. 2017. Secretory structures in Aldama species (Heliantheae-Asteraceae): morphology, histochemistry and composition of essential oils. Flora 228: 39-49.

BOTELHO MA, NOGUEIRA NAP, BASTOS GM, FONSECA SGC, LEMOS TLG, MATOS FJA, MONTENEGRO D, HEUKELBACH RAO VS \& BRITO GAC. 2007. Antimicrobial activity of the essential oil from Lippia sidoides, carvacrol and thymol against oral pathogens. Braz J Med Biol Res 40: 349-356. 
BRAND-WILLIAMS W, CUVELIER ME \& BERSET C. 1995. Use of a free radical method to evaluate antioxidant activity. Lebensm Wiss Technol 28: 25-30.

CHANG R, MORAIS SAL, DUARTE KC, GUZMAN VB \& NASCIMENTO EA. 2011. A new approach for quantifying furanodiene and curzerene. A case study on the essential oils of Eugenia uniflora (pitangueira) leaves. Braz I Pharmacogn 21(3): 392-396.

COSTA DP, ALVES FILHO EG, SILVA LMA, SANTOS SC, PASSOS XS, SILVA MRR, SERAPHIN JC \& FERRI PH. 2010. Influence of fruit biotypes on the chemical composition and antifungal activity of the essential oils of Eugenia uniflora leaves. J Braz Chem Soc 21(5): 851-858.

COSTA DP, SANTOS SC, SERAPHIN JC \& FERRI PH. 2009. Seasonal variability of essential oils of Eugenia uniflora leaves. J Brazil Chem Soc 20(7): 1287-1293.

DINIZ MER \& BUSCHINI MLT. 2016. Diversity of flower visiting bees of Eugenia uniflora L. (Myrtaceae) in fragments of Atlantic Forest in South Brazil. Sociobiology 63(3): 982-990.

FERREIRA TP, KUCKELHAUS IGP, TSCHOEKE PH, CANGUSSU AS, BORGES JCM, MOURA WS \& AGUIAR RWS. 2017. Influence of seasonality on the yield and composition of the essential oil of Siparuna guianensis Aublet. Afr J Biotechnol 16(29): 1611-1618.

GALHIANE MS, RISSATO SR, CHIERICE GO, ALMEIDA MV \& SILVA LC. 2006. Influence of different extraction methods on the yield and linalool content of the extracts of Eugenia uniflora L. Talanta 70: 286-292.

GALLUCCI S, PLACERES-NETO A, PORTO C, BARNIZAN D, COSTA I, MARQUES K, BENEVIDES P \& FIGUEIREDO R. 2010. Essential oil of Eugenia uniflora L.: an industrial perfumery approach. J Essent Oil Res 22: 176-179.

GHAZOUANI N, SIFAOUI I, BACHROUCH O, ABDERRABBA M, PINERO JE \& LORENZO-MORALES J. 2017. Essential oil composition and anti Acanthamoeba studies of Teucrium ramosissimum. Exp Parasitol 183: 207-211.

GRIFFIS JL \& MANNES MM. 2005. Inheritance of fruit color in Surinan Cherry (Eugenia uniflora L.) - A preliminary study. Proc Fla State Hort Soc 118: 198-199.

HAMDI OAA, YE LJ, KAMARUDIN MNA, HAZNI H, PAYDAR M, LOOI CY, SHILPI JA, KADIR HA \& AWANG K. 2015. Neuroprotective and antioxidant constituents from Curcuma zedoaria rhizomes. Rec Nat Prod 9(3): 349-355.

KUMAR AS, JEYAPRAKASH K, CHELLAPPAN DR \& MURUGAN R. 2017. Vasorelaxant and cardiovascular properties of the essential oil of Pogostemon elsholtzioides. J Ethnopharmacol 199: 86-90.

LAGO JHG ET AL. 2011. Chemical and biological evaluation of essential oils from two species of Myrtaceae
- Eugenia uniflora L. and Plinia trunciflora (O. Berg) Kausel. Molecules 16: 9827-9837.

LIU Y, WANG W, FANG B, MA F, ZHENG Q, DENG P, ZHAO S, CHEN M, YANG G \& HE G. 2013. Anti-tumor effect of germacrone on human hepatoma cell lines through inducing G2/M cell cycle arrest and promoting apoptosis. Eur J Pharmacol 698: 95-102.

OGUNWANDE IA, OLAWORE NO, EKUNDAYO O, WALKER TM, SCHMIDT JM \& SETZER WN. 2005. Studies on the essential oils composition, antibacterial and cytotoxicity of Eugenia uniflora L. Int J Aromather 15: 147-152.

OLIVEIRA AL, LOPES RB, CABRAL FA \& EBERLIN MN. 2006. Volatile compounds from pitanga fruit (Eugenia uniflora L.). Food Chem 99: 1-5.

PACHECO-SILVA NV \& DONATO AM. 2016. Morpho-anatomy of the leaf of Myrciaria glomerata. Rev Bras Farmacogn 26: $275-280$.

PEREIRA CB, KANUNFRE CC, FARAGO PV, BORSATO DM, BUDEL JM, MAIA BHLNS, CAMPESATTO EA, SARTORATTO A, MIGUEL MD \& MIGUEL OG. 2017. Cytotoxic mechanism of Baccharis milleflora (Less.) DC. Essential oil. Toxicology in Vitro 42: 214-221.

R CORE TEAM. 2014. R: A language and environment for statistical computing. R Foundation for Statistical Computing, Vienna, Austria.

RETAMALES HA \& SCHARASCHKIN T. 2015. Comparative leaf anatomy and micromorphology of the Chilean Myrtaceae: Taxonomic and ecological implications. Flora 217: $138-154$

REZENDE WP, BORGES LL, ALVES NM, FERRI PH \& PAULA JR. 2013. Chemical variability in the essential oils from leaves of Syzygium jambos. Braz J Pharmacogn 23(3): 433-440.

RODRIGUES KAF, AMORIM LV, OLIVEIRA JMG, DIAS CN, MORAES DFC, ANDRADE EHA, MAIA JGS, CARNEIRO SMP \& CARVALHO FAA. 2013. Eugenia uniflora L. essential oil as a potential antiLeishmania agent: effects on Leishmania amazonensis and possible mechanisms of action. Evidence-Based Complem. Alter Med 1-10. doi: 10.1155/2013/279726.

SANTOS FR, BRAZ-FILHO R \& CASTRO RN. 2015. Influência da idade das folhas de Eugenia uniflora L. na composição química do óleo essencial. Quím Nova 38(6): 762-768.

SANTOS JFS ET AL. 2018. Chemical composition, antifungal activity and potential anti-virulence evaluation of the Eugenia uniflora essential oil against Candida spp. Food Chem 261: 233-239.

SILVA ALG \& PINHEIRO MC. 2007. Biologia floral e da polinização de quatro espécies de Eugenia L. (Myrtaceae). Acta Bot Bras 21(1): 235-247. 
SILVA FAZ \& AZEVEDO CAV. 2016. The Assistat Sftware Version 7.7 and its use in the analysis of experimental data. Afr J Agric Res 11(39): 3733-3740.

SILVA VP ET AL. 2018. Chemical composition and in vitro leischmanicidal, antibacterial and cytotoxic activities of essential oils of the Myrtaceae family occurring in the Cerrado biome. Ind Crop Prod 123: 638-645.

SOUSA RMF ET AL. 2015. Chemical composition, cytotoxic, and antibacterial activity of the essential oil from Eugenia calycina Cambess. leaves against oral bactéria. Ind Crop Prod 65: 71-78.

STEFANELlO MEA, PASCOAL ACRF \& SALVAdOR MJ. 2011. Essential oils from Neotropical Myrtaceae: chemical diversity and biological properties. Chem Biodivers 8: 73-94.

STESEVIC D, BOZOVIC M, TADIC V, RANCIC D \& SREVANOVIC DZ. 2016. Plant-part anatomy related composition of essential oils and phenolic compounds in Chaerophyllum coloratum, a Balkan endemic species. Flora 220: 37-51.

THADEO M, MEIRA RMSA, AZEVEDO AA \& ARAÚJO JM. 2009. Anatomia e histoquímica das estruturas secretoras da folha de Casearia decandra Jacq. (Salicaceae). Rev Brasil Bot 32(2): 329-338.

THEANPHONG O, MINGVANISH W \& KIRDMANEE C. 2015. Chemical constituents and activities of essential oil from Curcuma aeruginosa Roxb. rhizome. Bull Health Sci Technol 13(1): 6-16.

VAN DEN DOOL H \& KRATZ PD. 1963. A generalisation of the retention index system including linear temperature programmed gas $3 / 4$ liquid chromatography. J Chromatogr 11: 463-471.

VERNA N \& SHUKLA S. 2015. Impact of various factors responsible for fluctuation in plant secondary metabolites. J Appl Res Med Aromat Plants 2: 105-136.

VICTORIA FN, LENARDÃO EJ, SAVEGNAGO L, PERIN G, JACOB RG, ALVES D, SILVA WP, MOTTA AS \& NASCENTE PS. 2012. Essential oil of the leaves of Eugenia uniflora L.: Antioxidant and antimicrobial properties. Food Chem Toxicol 50: 2668-2674.

WANG W, WU N, ZU YG \& FU YJ. 2008. Antioxidative Activity of Rosmarinus officinalis L. essential oil to its main components. Food Chem 108: 1019-1022.

ZHAO J, ZHANG J, YANG B, LV G \& LI S. 2010. Free radical scavenging activity and characterization of sesquiterpenoids in four species of Curcuma using a TLC bioautography assay and GC-MS analysis. Molecules 15: 7547-7557.

ZHONG ZF, QIANG WA, WANG CM, TAN W \& WANG YT. 2016. Furanodiene enhances the anti-cancer effects of doxorubicin on ER $\alpha$-negative breast cancer cells in vitro. Eur J Pharmacol 774: 10-19.

ZOGHBIA MGB, GUILHON GMSP, SARGES FN, PEREIRA RA \& OLIVEIRA J. 2011. Chemical variability of the volatiles from the leaves of Eugenia protenta McVaugh (Myrtaceae) growing wild in the North of Brazil. Biochem Syst Ecol 39: $660-665$.

\section{How to cite}

CIPRIANO RR, MAIA BHLNS \& DESCHAMPS C. 2021. Chemical variability of essential oils of Eugenia uniflora L. genotypes and their antioxidant activity. An Acad Bras Cienc 93: e20201299. DOI 10.1590/00013765202120181299.

Manuscript received on December 10, 2018;

accepted for publication on June 27, 2019

\section{ROGER R. CIPRIANO ${ }^{1}$}

https://orcid.org/0000-0002-3896-9074

\section{BEATRIZ H.L.N.S. MAIA ${ }^{2}$}

https://orcid.org/0000-0001-5896-2892

\section{CÍCERO DESCHAMPS ${ }^{1}$}

https://orcid.org/0000-0003-0786-0532

${ }^{1}$ Programa de Pós-Graduação em Agronomia/Produção Vegetal, Universidade Federal do Paraná, Setor de Ciências Agrárias, Departamento de Fitotecnia e Fitossanitarismo, Rua dos Funcionários, 1540, Juvevê, 80035-050 Curitiba, PR, Brazil ${ }^{2}$ Programa de Pós-Graduação em Química, Universidade Federal do Paraná, Setor de Ciências Exatas, Departamento de Química, Centro Politécnico, Av. Cel. Francisco H. Santos, 100, Jardim das Américas, 81531-980 Curitiba, PR, Brazil

Correspondence to: Roger Raupp Cipriano

E-mail: rogerraupp@gmail.com

\section{Author contributions}

All authors contributed significantly to elaboration of this manuscript: Roger R. Cipriano conducted the collection of plant material extraction and analysis of essential oils. Beatriz H.L.N.S. Maia helped in the analysis of essential oils. Cicero Deschamps supervised the estudy and contributed to writing of de manuscript.

\section{(cc) BY}

